\title{
Carnets
}

Revue électronique d'études françaises de l'APEF

Deuxième série - 5 | 2015

Imaginaires de guerre et autres conflits

\section{De l'oubli à la reconnaissance}

Le récit de guerre mis en images dans La Nueve. Les espagnols qui ont libéré Paris de Paco Roca

\section{Vanessa Auroy}

\section{OpenEdition}

1 Journals

\section{Édition électronique}

URL : http://journals.openedition.org/carnets/463

DOI : $10.4000 /$ carnets. 463

ISSN : 1646-7698

Éditeur

APEF

\section{Référence électronique}

Vanessa Auroy, "De l'oubli à la reconnaissance », Carnets [En ligne], Deuxième série - 5 | 2015, mis en ligne le 30 novembre 2015, consulté le 19 avril 2019. URL : http://journals.openedition.org/ carnets/463 ; DOI : 10.4000/carnets.463

Ce document a été généré automatiquement le 19 avril 2019

\section{(c) (i) (9)}

Carnets est mis à disposition selon les termes de la licence Creative Commons - Atribution - Pas d'utilisation commerciale 4.0 International. 


\section{De l'oubli à la reconnaissance}

Le récit de guerre mis en images dans La Nueve. Les espagnols qui ont libéré Paris de Paco Roca

Vanessa Auroy

1 Depuis peu, une nouvelle « mouvance artistique » a fait son apparition dans la littérature espagnole: la bande dessinée. Peu utilisée jusqu'alors pour évoquer les conflits, sa production, sa diffusion et surtout son impact sur l'imaginaire du lecteur sont aujourd'hui indubitables. Il suffit de regarder les catalogues des librairies ou les références des sites Internet pour se rendre compte de la prolixité dans ce domaine. Des auteurs de plus en plus nombreux et des listes de titres d'ouvrages s'affichent alors sous les yeux du consultant : 36-39 Malos tiempos de Carlos Giménez, El arte de volar de Antonio Altarriba et Kim, El ángel de la retirada de Paco Roca ne sont que quelques-uns des exemples possibles.

Quant à l'œuvre de Paco Roca, Los surcos del azar parue en 2013, elle est très vite devenue un succès de librairie en Espagne comme en France. À travers ce récit en bandes dessinées, nous suivons l'itinéraire chaotique et véridique de réfugiés espagnols en France. Ces militants anarchistes et communistes de l'Espagne républicaine vaincue ont intégré la résistance française en incorporant en nombre et de façon surprenante, la neuvième compagnie du colonel Leclerc qui a libéré la capitale parisienne, d'où la traduction évocatrice du titre de la bande dessinée en français : La Nueve. Les républicains espagnols qui ont libéré Paris.

3 Paco Roca met ainsi à l'honneur des hommes qui ont dépassé les réticences que pouvaient leur apporter leurs convictions idéologiques particulières pour défendre la liberté des Hommes, même s'ils n'avaient pas réussi à empêcher la mainmise franquiste sur leur pays. L'auteur espagnol évoque aussi en leur redonnant une apparence, une vie, une image aux yeux du lecteur, des hommes oubliés et qui ont libéré la capitale de la France le 24 août 1944. Seules quelques cérémonies et commémorations discrètes ont été célébrées depuis cet événement marquant de l'Histoire française. D'ailleurs, la première cérémonie d'ampleur pour célébrer ces héros de la résistance et à laquelle ont assisté les autorités politiques et militaires n'a eu lieu que le 24 août 2014.

4 À travers l'analyse de l'ouvrage de Paco Roca nous nous proposons donc de montrer comment une œuvre artistique peut rendre la parole et l'existence à des combattants 
pour la liberté oubliés du grand public, héros, martyrs et traîtres à leurs valeurs par intermittence. Il s'agit aussi de montrer comment les mots mais surtout les images représentent les horreurs, les douleurs autant physiques que morales des guerres quelles qu'elles soient.

\section{La genèse de l'œuvre}

5 Bien que le thème de la Guerre civile espagnole ait produit une abondante littérature de fiction et que celui de la Seconde Guerre Mondiale et de la résistance ait été encore plus propice aux créations littéraires et cinématographiques, le rôle joué par les républicains espagnols réfugiés en France n'a été que peu évoqué durant les 70 dernières années. Depuis l'approbation de la Loi de Mémoire historique, fin 2007, les descendants des combattants républicains de la Guerre civile espagnole ont cherché à réhabiliter les actions de leurs parents et grands-parents pour défendre la liberté. L'ouverture complète des archives en 1999 a aussi permis aux historiens de mettre à jour les actes, les héros de cette guerre mais aussi ses épisodes plus dramatiques. Ainsi, les Républicains ayant perdu cette guerre, beaucoup ont fui pensant mieux revenir plus tard afin d'éradiquer à jamais le Franquisme de la Péninsule ibérique. Ils se sont notamment réfugiés en France et ont été «accueillis » dans des camps de concentration dans le sud: Argelès sur mer, Saint Cyprien, Gurs... Les conditions de vie y étaient terribles, dénuées de toute hygiène, les Espagnols survivaient dans des bâtiments insalubres, abandonnés par les autorités françaises.

6 Peu d'auteurs et d'historiens se sont penchés sur cet épisode de l'Histoire. Les livres y faisant référence sont longtemps restés rares et les témoignages aussi. Quant à savoir ce qu'il est advenu de ces hommes et de ces femmes lorsque la guerre contre le nazisme a pris de l'ampleur et que ces réfugiés n'étaient plus une préoccupation pour les autorités, cela ne fut pas la priorité des chercheurs. Le fait que beaucoup d'entre eux aient intégré la résistance française a donc été occulté. Sous prétexte de lutte pour la liberté, il n'y avait plus de nationalité à revendiquer, comme le souligne Evelyn Mesquida, qui s'est intéressée aux Espagnols qui ont intégré la Seconde Division Blindée du Général Leclerc et qui ont été primordiaux dans la libération de Paris :

La politique et l'historiographie française ont oublié et minimisé la participation de ces hommes dans la lutte contre la barbarie. Aujourd'hui encore, des historiens de renom continuent d'ignorer dans leurs travaux la présence importante des combattants espagnols au sein des forces françaises pendant le Seconde Guerre Mondiale.

Peu à peu, cependant, l'on découvre qu'ils ne furent pas une "poignée ", ni " quelques-uns », ni « quelques soldats » à intégrer ces forces, mais bien des milliers et des milliers d'hommes, sans patrie et disposés à continuer la lutte pour la liberté. Plusieurs milliers d'entre eux perdirent la vie dans ce combat. (Mesquida, 2008: 267)

7 Son ouvrage basé sur les témoignages de quelques rares survivants de cette division blindée a donc été le point de départ de Paco Roca pour l'écriture de sa bande dessinée comme il l'indique dans ses remerciements (Roca, 2013a : 318). 


\section{Redonner une vie et une parole}

8 Le prologue de l'ouvrage d'Evelyn Mesquida a été écrit par Jorge Semprun. Militant espagnol reconnu en Espagne et en France, auteur prolixe qui a lui aussi raconté de façon plus ou moins autobiographique sa fuite d'Espagne, Jorge Semprun connaissait l'histoire de ces combattants, en avait rencontré certains. Mais il savait aussi à quel point ces hommes étaient silencieux, incapables de raconter leur vie, de s'opposer au silence des autorités à leur sujet. C'est pourquoi il conclut le prologue par ces mots :

Je crois qu'il faudrait faire une histoire globale sur tous ces combattants. Il faut continuer à parler d'eux en cherchant des documents, en incitant les cinéastes à réaliser des films sur l'incroyable vie de ces hommes. L'histoire de la Nueve est un thème pour un grand film. (Mesquida, $2008: 9$ )

Ce ne sera pas un film qui rendra hommage aux combattants de la division Leclerc mais bien une bande dessinée. Ou plutôt, il s'agit ici d'un roman graphique. L'ouvrage de Paco Roca fait en effet plus de 300 pages et est très documenté. Paco Roca a beaucoup lu, a consulté des archives, a rencontré des historiens. Ce fut un travail de longue haleine car il mettra cinq ans à produire son ouvrage ce qui est extrêmement long selon ses dires. Il présente d'ailleurs son travail comme celui d'un historien ou d'un journaliste qui préparerait un documentaire. Il revendique aussi ses cautions intellectuelles. Le paratexte qui se retrouve à la fin dans les éditions française et espagnole est signé par Robert $\mathrm{S}$. Coale, historien à l'Université Paris III et spécialiste de l'Histoire contemporaine espagnole. La préface de l'édition française est rédigée par Anne Hidalgo, maire de Paris. Il y a donc une véritable volonté de rendre un hommage sérieux et honorable à ces hommes qui ont combattu. Malgré tout, Paco Roca ne parvient pas à entrer en contact avec les anciens combattants survivants souvent du fait de leur état de santé ou de leur grand âge.

Paco Roca se met aussi en scène dans sa bande dessinée. Jeune espagnol, il vient en France pour interviewer un compatriote ancien soldat de la seconde division blindée du Général Leclerc dans le but d'écrire un livre ou un film. Avant de rencontrer un certain Miguel Ruiz, il croise d'abord Albert, le garagiste qui connaît, pense-t-il, Miguel depuis plus de 10 ans mais qui ignore tout de cette partie de la vie du vieil homme : " Tu veux me faire croire que le papy a été un héros? Ha ha ha! Miguel héros de guerre, je ne peux pas y croire » (Roca, 2013a : 20). Albert va donc de surprise en surprise au fur et à mesure du récit que Miguel voudra bien confier à Paco.

D'autre part, si le personnage d'Albert, sans doute purement fictionnel, ne sait rien de la jeunesse de Miguel c'est aussi parce que ce dernier a tenté d'oublier ou en tout cas de ne pas se souvenir d'une partie de sa vie, assailli par le ressentiment: "J'ai quatre-vingtquatorze ans et la plupart de mes amis sont morts, sans aucune reconnaissance. Si pour vous, ce n'est pas trop tard » (Roca, 2013a: 21 et 42). Néanmoins, la présence de Paco et ses questions seront pour Miguel une catharsis salvatrice: «Merci (...) pour m'avoir permis de retrouver une partie d'une vie dont je n'osais plus me souvenir » (Roca, 2013a : 314). Le Miguel du roman graphique est plus ou moins inventé. Au début, il se présente comme Miguel Ruiz. Paco aurait obtenu des informations sur cet homme par un historien qui prétendait que Miguel Ruiz est en réalité Miguel Campos, combattant de la Nueve dont on perd la trace avant la fin de la Seconde Guerre Mondiale. Au moment de l'embarquement pour l'Angleterre, Miguel change donc de nom par peur des représailles 
contre lui et sa famille restée en Espagne, ce qui était vraisemblablement chose courante parmi les soldats. Dans la bande dessinée, Paco réagit au nom de Campos car il apparaît cité dans les mémoires de Raymond Dronne, capitaine de la Nueve. Et comme il le rajoute dans son interview, cette présentation et le peu d'informations sur Miguel permettent à l'auteur espagnol de «broder » [especular] sur son histoire et de créer ainsi une œuvre fictionnelle basée sur des faits et des personnes réelles.

\section{Mais qui étaient ces héros silencieux?}

Pour bien comprendre pourquoi ces hommes éprouvaient un tel ressentiment vis-à-vis de la France, il faut savoir qui ils étaient. Et même si l'ouvrage d'Evelyn Mesquida donne toutes les informations nécessaires à leur sujet, apporte une véracité aux faits, le roman graphique de Paco Roca met en images les événements et « redonne » une vie aux acteurs de la résistance.

Dès le début, le lecteur se rend compte que la scène a lieu sur un port en Espagne. Des hommes, des femmes et des enfants attendent un bateau pour fuir, encadrés par des miliciens républicains qui portent des uniformes reconnaissables. Paco Roca s'est beaucoup documenté afin de respecter dans le détail les tenues, les expressions de l'époque. Le silence ou le peu de paroles souligne aussi toute l'angoisse de ces personnes qui attendent d'être sauvées. Les vignettes, de couleurs sombres car la scène se passe de nuit, expriment la peur qui se voit sur les visages quand retentissent les bruits des tirs et des combats qui semblent se rapprocher. La bande dessinée permet ce que permet un film mais pas un roman, le silence des personnes. Le lecteur peut ainsi se focaliser sur l'attitude des personnages sur les traits qui leur sont donnés. Il peut, temporairement, essayer de se mettre à la place de ces personnes apeurées qui fuient, essayer d'éprouver les mêmes sentiments ou d'imaginer l'ambiance. L'on pourrait se dire que ceci est aussi possible avec un film. Mais le cinéaste est plus directif. Les films sont souvent plus bavards. Le spectateur se retrouve surpris lorsqu'il visionne un film avec peu de dialogues. De plus, les films qualifiés « de guerre » sont envahis par le bruit des armes et des canons. Au contraire, dans sa bande dessinée, Paco Roca évoque les combats par des onomatopées mais s'intéresse surtout dès le début aux personnes. D'ailleurs, dès la page 5 un personnage avec une capote est mis en évidence dans une vignette. Nous apprenons très vite qu'il s'appelle Miguel et supposons qu'il va devenir le protagoniste de cet ouvrage car il se détache des autres personnages. Il est présent dans pratiquement toutes les vignettes suivantes, comme s'il était suivi par une caméra qui zoomerait sur lui. Miguel est donc, selon son uniforme, un soldat de l'armée républicaine qui fuit l'Espagne en guerre. La scène se passe dans le port d'Alicante où le cargo anglais Stanbrook a accosté le 28 mars 1939 soit quatre jours avant la fin officielle de la guerre et la victoire franquiste. Le bateau accueillera à son bord près de 3000 réfugiés soit plus de trois fois sa capacité. Page 11, Paco Roca dessine l'amoncellement de personnes sur le quai et sur le bateau où certains hommes sont allés se réfugier jusque sur les mâts. Le dessinateur espagnol n'a pas hésité à montrer toute la détresse de ceux qui n'ont pu monter sur le bateau en mettant en scène, encore une fois sans paroles, un homme résigné et d'apparence tranquille qui s'égorge sous le regard incrédule des autres restés eux aussi à terre (Roca, 2013a : 29). Les souffrances morales des réfugiés commencent ici en voyant ces milliers de personnes dans l'impossibilité d'embarquer et qui seront sans doute soumises à une mort prochaine. 
14 Après, ce ne fut qu'une suite d'humiliations. La traversée pénible se fait dans le froid, sans nourriture (Roca, 2013a: 28). Arrivés à Oran, il leur est impossible de débarquer. Le gouvernement n'autorise que les personnes ayant des papiers en règle à poser le pied sur le territoire français. Or, tous ont fui les combats en n'emportant que le strict nécessaire voire aucune affaire. Ils se retrouvent donc coincés sur le cargo à survivre dans des conditions déplorables, envahis par les poux, sans rien à manger, ravitaillés par les habitants de la ville de façon plus ou moins clandestine, subissant les intempéries sans pouvoir s'abriter. La promiscuité et l'hygiène inexistante obligent même ces hommes à se comporter comme des animaux et à faire leurs besoins depuis le bastingage, sans aucune intimité ni décence (Roca, 2013a: 43 à 51). Le traitement inhumain infligé aux réfugiés espagnols a bien entendu eu des conséquences dramatiques sur leur état d'esprit comme l'explique Miguel à Paco :

-Vous êtes restés encore longtemps à bord?

- Pratiquement un mois. Dans des conditions lamentables. Certains d'entre nous

sont devenus fous. D'autres se sont suicidés. (Roca, 2013a : 53)

L'humiliation ne s'arrête pas là. Les soldats républicains peuvent choisir de rentrer en Espagne (pays qu'ils avaient fui et qui est maintenant sous régime franquiste) ou d'intégrer la Légion étrangère ou encore d'aller dans les camps de travail pour les réfugiés. Pensant être accueillis comme de véritables réfugiés de guerre, de nombreux espagnols choisissent les camps. Miguel est envoyé au camp Morand comme beaucoup d'entre eux. Ce camp se trouve en plein désert tunisien, le trajet se fait en wagon à bestiaux. L'accueil offert par les militaires français est très loin de ce qu'ils pouvaient imaginer :

- Des Espagnols! Comme vous devez le savoir les colonies françaises d'Afrique se sont ralliées au gouvernement du maréchal Pétain... Dès cet instant vous perdez votre statut de réfugiés. A moi, on ne la fait pas, vous êtes des rebuts, des rouges, et la France ne nourrira pas gratuitement des antifascistes. Vous allez avoir, ici, l'occasion de faire quelque chose d'utile. Vous allez participez à la construction de la voie ferrée transsaharienne. Si vous travaillez, vous mangez. Si vous ne le faites pas, vous êtes punis.

- Messieurs les rouges, vous ne partirez d'ici que les pieds devant. (Roca, 2013a : 65)

Miguel et ses compatriotes qui avaient fui l'Espagne franquiste se retrouvent donc punis en territoire français pour leurs convictions politiques et leur antifascisme ce qui ne fera que renforcer un fort ressentiment qui réapparaitra régulièrement et qui durera jusqu'en 2013 pour le personnage de Miguel : «Pendant combien de temps allons-nous être punis pour avoir défendu la liberté » (Roca, 2013a : 66). Ils deviennent des ennemis de la France de Pétain. Ils sont donc traités comme tels dans les camps en étant prisonniers et en travaillant en pleine chaleur dans le désert, punis et fouettés en cas de rébellion ou lorsqu'ils revendiquent en chantant leur antifascisme. Les conditions auxquelles ils sont soumis s'apparentent grandement aux conditions dans les camps de travail allemands et non à celles qui pourraient être attendues dans les camps de réfugiés.

\section{De libertaires à militaires}

17 En 1942, les troupes alliées débarquent en Afrique et libèrent les camps. Épuisés par un traitement qu'ils jugent inhumain et désirant à nouveau combattre le fascisme afin de rapidement aller libérer l'Espagne du joug franquiste, de nombreux réfugiés espagnols notamment anarchistes et communistes demandent à intégrer les Corps Francs d'Afrique 
(CFA). Il s'agit d'une armée parallèle qui se forme au Maghreb suite à l'appel du Général De Gaulle. Composés principalement de résistants au régime de Vichy, ces corps sont commandés par le Général Henri Giraud. Ils s'illustrent lors des combats contre les troupes de Mussolini et l'Afrikakorps allemand et lors de la bataille de Bizerte en 1943 (Roca, 2013a : 96 à 117). Mais là encore l'accueil est loin d'être cordial pour les Espagnols. Les hommes acceptent de combattre mais veulent le faire sous drapeau républicain. Bien entendu, les autorités françaises s'y opposent dans un premier temps. Ce désir va pourtant persister tout au long des années de combats de ces soldats comme une revendication de leurs opinions et de leurs convictions. L'armée française exprime une autre réticence à l'égard de ces hommes: "L'armée française n'a pas besoin dans ses rangs d'hommes qui ont déjà perdu une guerre » (Roca, 2013a : 87). Pourtant, la guerre civile espagnole, bien que perdue, aura été pour eux un terrain d'entraînement aux combats.

En 1943, le Général Leclerc reçoit l'ordre de former la Deuxième Division Blindée (que nous nommerons désormais $2^{2}$ ème $\mathrm{DB}$ ) suite à la réorganisation de l'armée française. Elle réunit les Forces Françaises Libres et l'Armée d'Afrique (CFA). L'infanterie mécanisée de la Division est composée par les Régiments de Marche du Tchad. Ces régiments sont essentiellement alimentés par les régiments de Tirailleurs sénégalais du Tchad et des hommes basés en Afrique du Nord qui se sont ralliés à la France Libre. Le $3^{\text {ème }}$ Régiment est dirigé par le commandant Joseph Putz à qui Miguel voue une grande estime (Roca, 2013a : 113), sous les ordres duquel il était déjà au sein des CFA et qui plus est, est un ancien brigadiste. Ce régiment est à son tour composé de compagnies, c'est-à-dire de regroupements d'hommes. Celles du $3^{\text {ème }}$ Régiment sont la Neuvième, la Dixième et la Onzième compagnie plus une compagnie d'appui. Miguel et ses camarades sont incorporés à la $9^{\text {ème }}$ qui est tout de suite appelée la Nueve ou le «Batallón hispano » car sur les 160 soldats qui la composent, 146 sont espagnols. Tous ou presque parlent espagnol : « (...) même le clairon sonnait le réveil matinal en espagnol » (Mesquida, 2008 : 107). Il faut donc à cette compagnie un capitaine maîtrisant l'espagnol. Ce sera le capitaine Raymond Dronne car sa femme est professeur d'espagnol et il parle un peu cette langue puisqu'il fut déjà cantonné en Espagne (Roca, 2013a : 137).

Pourtant, ce point sera le moindre de ses soucis. En effet, les hommes sont indisciplinés et n'acceptent pas les ordres. Là encore leur tempérament impulsif, leur désir de revanche et leurs convictions prennent le dessus fréquemment (Roca, 2013a: 93-94). Dronne va jusqu'à s'en plaindre au Général Leclerc :

-...Ils sont indisciplinés, Général.

- Mais ce sont de bons soldats. Des hommes vaillants ayant une grande expérience du combat. Nous avons besoin d'hommes comme eux dans notre division.

- Ces espagnols n'acceptent pas les ordres.

- Ils ne les acceptent que de ceux qu'ils respectent. Putz qui les a eus sous ses ordres, assure que, quand ils vous auront accordé leur confiance, ils vous suivront jusqu'à la mort. Car vous, ils vous respectent.

- Pourquoi moi, Général?

- Ils n'exécuteront jamais d'ordres venant d'un Vichyste qui a retourné sa veste. Et ils savent que, depuis le début, vous avez lutté pour la France Libre (...) (Roca, 2013a: 136-137)

Il saura effectivement gagner leur confiance et même s'ils ne le suivent pas tous jusqu'à la mort, ils iront avec lui jusqu'à Paris. D’ailleurs, dans ses mémoires, Dronne parlera de ces/ ses hommes en termes élogieux: «Dans leur grande majorité, ils n'avaient pas l'esprit 
militaire, ils étaient même antimilitaristes, mais ils étaient de magnifiques soldats, des guerriers vaillants et expérimentés » (Mesquida, $2008: 108) .70$ ans après, ce lien entre les combattants et leur hiérarchie militaire est encore présent alors qu'il paraissait improbable. Rafael Gómez, l'un des soldats survivants de la Nueve, mais qui n'apparaît pas dans la bande dessinée, était présent aux commémorations du 24 août 2014. Il n’a pu retenir ses larmes et son émotion face à l'hommage tant civil que militaire qui lui était rendu, à lui et à ses camarades morts au combat, en remerciement pour leur dévouement pour la libération de Paris.

21 Avec la création de la $2^{\text {ème }} \mathrm{DB}$, les soldats reçoivent un nouvel équipement fourni par les Américains et auquel ils vont devoir s'habituer. Des formations sont mises en place pour apprendre à manier les fusils notamment. Jusqu'alors les soldats n'avaient utilisé que des armes datant de la Première Guerre Mondiale que ce soit lors des combats en Afrique du Nord ou durant la Guerre Civile espagnole. De plus, n'étant pas des militaires professionnels, les hommes ne connaissent pas les tactiques à suivre pour combattre en sacrifiant le moins de vies possible. Cet enseignement dispensé par les Américains ou par des hommes formés par eux sera l'occasion de vives tensions (Roca, 2013 : 142). Les EtatsUnis pourvoient aussi la compagnie en véhicules car elle fait partie d'une division motorisée et blindée. La Nueve se fera aussi connaître grâce à ses half-tracks, autochenilles pouvant transporter jusqu'à 10 combattants. Ces véhicules étaient maniables et permettaient des assauts rapides et efficaces. Certains, notamment ceux de la Nueve, étaient équipés de mitrailleuses et de canons. La formation fut intense. Le Général Leclerc voulait revenir en Europe avec des hommes formés, responsables et aguerris :

Organisée selon le modèle des unités des forces américaines, Leclerc commença la formation en prenant comme base les unités avec le niveau le plus haut, pour les niveler vers le haut, demandant à ses hommes qu'ils résolvent les difficultés quotidiennes comme ils le feraient dans le cadre de cette nouvelle armée moderne, en respectant la mission reçue et en tenant compte que l'armée blindée était devenue une arme rapide, une arme difficile et qu'ils devraient prendre des initiatives rapides étant donné qu'ils n'auraient pas le temps de se familiariser avec l'ennemi. Pour le soldat de cavalerie qu'était Leclerc, un cheval valait autant que son cavalier, et dans cette nouvelle armée, le véhicule valait ce que valait son équipage. (Mesquida, 2008 : 105)

Mais, même si les miliciens de la République espagnole deviennent des soldats disciplinés, adroits dans le maniement des armes et tacticiens, ils n'en restent pas moins des anarchistes et des communistes encore réticents à l'autorité militaire. Ainsi, lorsqu'il faut baptiser les véhicules comme il est de tradition avant de partir au combat, les vieilles querelles politiques ressurgissent comme le montre la conversation entre le commandant Putz et le capitaine Dronne :

[Dronne] : Vous connaissez les espagnols...Ils n'ont pas réussi à se mettre d'accord. Certains veulent utiliser des noms de héros anarchistes, d'autres de socialistes et d'autres de communistes.

[Putz]: Non, non! Leclerc ne veut pas de politique. Qu'ils choisissent des noms qui ne posent pas de problèmes. Des noms de villes d'Espagne par exemple. (Roca, 2013a: 143)

C'est ce que feront les hommes en nommant leurs véhicules Guadalajara, Teruel, Madrid, Belchite...mais avec une arrière-pensée comme le dit amusé Miguel à Paco :

[Dronne] : Ce sont des noms de villes...

[Paco]: Celles où ils sont nés? 
[Miguel] : C'étaient les noms des villes où les républicains avaient combattu Franco.

[Dronne] : Si Leclerc pose la question dites-lui que c'est votre lieu de naissance. Et

pour ma jeep, trouvez quelque chose de moins compromettant. (Roca, 2013a : 143)

Ils la baptiseront « Mort aux cons », non par goût de la provocation pour une fois mais car cette expression était souvent utilisée par le capitaine Dronne quand il se mettait en colère selon les témoignages.

\section{Les grandes manœuvres commencent}

Les hommes de la Nueve sont prêts à faire route pour l'Europe. Commence alors leur éphémère légende. Hommes et véhicules embarquent pour se rendre en Angleterre où se trouve le Général De Gaulle et où Leclerc vient d'arriver. Durant le trajet, non loin des côtes espagnoles, certains dont Miguel ont encore la certitude qu'une fois la France libérée du nazisme ce sera le tour de l'Espagne (Roca, 2013a : 158). Ils y restent cantonnés trois mois. Puis, c'est le départ pour la France. L'arrivée sur le territoire français est emplie d'émotion. En effet :

Pour la majorité des soldats de la division, la distance jusqu'aux côtes françaises se comptait surtout en temps...quatre ans...quatre longues années. Le premier geste de beaucoup d'entre eux en débarquant fut de ramasser une poignée de sable. Certains pleuraient. La Division Leclerc était la première troupe française qui débarquait sur le sol français depuis quatre ans. L'émotion gagna aussi de nombreux Espagnols. (...) La France était pour eux à ce moment la répétition d'un prochain « débarquement » dans leur pays. (Mesquida, $2008: 133$ )

Paco Roca met en scène ce débarquement et les attitudes de chacun pages 178 à 180 et termine sa reconstitution par l'accueil chaleureux que les Français éprouvés par la guerre leur ont accordé croyant avoir affaire uniquement à des soldats français. Mais ce temps de joie sera de courte durée. La $2^{\text {ème }}$ DB est placée sous le commandement du Général Patton et des Américains.

Leclerc est un patriote ambitieux. Il veut avancer rapidement sur Paris afin d'être le premier à y entrer et libérer la capitale. La Nueve sera en première ligne car la compagnie a une expérience des combats rapprochés. Elle est donc envoyée régulièrement en reconnaissance. Ainsi, les unités de la Wehrmacht se trouvent en nombre à Écouché, dans l'Orne, sur la route de Paris. Elles occupent notamment un château dans lequel les troupes allemandes retiennent prisonniers des soldats américains. La Nueve prend d'assaut le château. Le combat est terrible. 150 allemands sont faits prisonniers. De nombreux soldats de la Nueve meurent lors de cette attaque (Mesquida, 2008: 136). Le traitement qu'a fait Paco Roca de cet épisode dans sa bande dessinée souligne toute l'ambiguïté qui peut exister entre réalité et fiction dans ce type d'œuvre. Le récit de la prise du château et les images de combats rapprochés apparaissent à la fois héroïques et mis en scène d'une façon digne des plus grands films de guerre qui ont vraisemblablement influencé Paco Roca comme il le dit dans son interview accordée aux éditions Delcourt. La violence n'est donc sans doute pas fictive. En revanche, les paroles échangées entre le soldat SS mortellement blessé et Miguel et son acolyte sont sans doute inventées car il y a très peu de témoignages des rares survivants de la Nueve (16 après la libération de Paris). De plus, Paco Roca n'a jamais pu rencontrer Miguel puisqu'il a disparu. Le fait d'inventer une vie à cet homme permet aussi à Paco Roca de justifier son choix de mettre en avant des hommes qui ont été violents et qui ont tué. En effet, sous l'apparence d'une conversation amicale dans la cuisine, Paco se permet de juger Miguel pour avoir tué des hommes de 
sang-froid, comme pourrait le faire tout à chacun 70 ans après les faits. La réaction de Miguel est épidermique et sans doute identique à ce qu'elle aurait pu être si la question lui avait été posée juste après la prise du château. Il se justifie en réaffirmant que cet événement s'est passé en temps de guerre et que ces hommes en avaient fait de même, la vengeance était donc légitime (Roca, 2013a : 215), d'où sa réticence à poursuivre la conversation : «Et pour qui tu te prends pour te permettre de me juger? Tu viens remuer la merde pour écrire un bouquin ou ce que tu crois être un bouquin!»

Ce ressentiment se retrouve tout au long de la bande dessinée car Miguel représente ces hommes qui ont lutté certes contre le nazisme mais aussi contre toute forme de fascisme bien avant le début de la Seconde Guerre Mondiale. Pourtant, ils souffrirent d'un manque de reconnaissance pour leurs actes héroïques car ils ont permis le rétablissement de la liberté durant toute leur vie. C'est donc un plaidoyer contre l'intolérance que Paco Roca met dans la bouche de Miguel en nuançant, non pas le propos mais les actes qui permettent de lutter. Les paroles de Miguel soldat de la Nueve prêt à exécuter des soldats nazis et celles du Miguel des années 2000 sont pourtant empreintes de la même conviction :

(...) les allemands sont la cause de toutes nos souffrances, de la Guerre d'Espagne, de notre captivité en Afrique, de cette guerre-ci! Tous ceux qui portent cet uniforme sont coupables. Pour cette raison, les Espagnols avaient la réputation de soldats sanguinaires. En réalité, quiconque avait une idée aussi claire du nazisme que nous aurait agi de la même façon. Pourtant, il y avait des moments où je doutais. Et avec les années, la haine et le désir de vengeance s'étant éteints, je regrette certains de mes actes. (...) Mais si c'était à refaire, je le referais pour barrer la route au nazisme. (Roca, 2013a : 222-223)

\section{La fin de l'aventure}

Si la Nueve est la première compagnie à entrer dans Paris, c'est grâce à sa réputation. Elle est envoyée par Leclerc et De Gaulle en avant-garde. Pourtant, la libération de Paris n'est pas un objectif militaire. Les Américains voulant arriver le plus rapidement possible en Allemagne afin de contenir les troupes nazies ont l'intention de contourner la capitale, la bataille pour la prise de la ville s'annonçant trop longue et aléatoire quant à son issue (Mesquida, 2008: 139). De Gaulle ne veut pas que Paris soit libéré par les communistes qui se soulèvent mais souhaite tout de même une libération par les Français (non les Américains). Pour Leclerc, grand tacticien, l'objectif diffère un peu. Il pense arriver plus vite en Allemagne (voire avant les troupes américaines) en coupant par la capitale. Le 23 août la $2^{2 \mathrm{ème}}$ DB se met donc en marche sous les yeux incrédules des Américains (Roca, 2013a : 256). Après avoir passé de multiples embuscades, ils arrivent dans la banlieue parisienne. Le 24 août au matin les troupes entrent dans Paris direction la préfecture où sont retranchés les insurgés et les FFI (Forces Françaises de l'Intérieur). Le half track Guadalajara dans lequel se trouve Miguel et ses acolytes est à la tête de la colonne. C'est l'un des derniers véhicules rescapés de cette journée historique et qui fut présenté au public lors des célébrations du 24 août 2014. Au passage de la Nueve la population envahit les rues et se précipite sur les soldats pour les embrasser croyant avoir affaire à des Français qui viennent les libérer (Roca, 2013a : 261).

Paco Roca prend alors le parti de réduire au maximum les dialogues et de privilégier le dessin. S'en suivent des pages de vignettes représentant Paris de nuit entrecoupées ou agrémentées d'un plan des rues de la ville sur lequel l'on peut suivre le trajet exact de la 
compagnie depuis la porte d'Italie jusqu'à l'Hôtel de ville: rue de la Vistule, rue Baudricourt, rue Nationale, rue Esquirol, boulevard de l'Hôpital, pont d'Austerlitz, quai Henri IV, quai des Célestins, Hôtel de ville. Le parcours se passe dans un calme relatif, sans coups de feu ni combats. Dronne et sa compagnie sont chaleureusement accueillis par le Comité National de la Résistance. Paco Roca fait même référence à la présence d'un journaliste du quotidien Libération qui aurait écrit un article publié quelques jours plus tard. Miguel Ruiz le conserve soigneusement parmi ses souvenirs car il est « (...) la preuve du long chemin parcouru par les Espagnols en exil» (Roca, 2013a: 268). En effet, ce document est extrêmement précieux car le protagonisme des Espagnols dans la libération de Paris sera vite oublié. D'ailleurs, cet événement est rapidement évoqué par Paco Roca lui-même puisqu'il n'occupe qu'une dizaine de pages sur un roman graphique qui en compte plus de 300 .

31 Puis, l'auteur espagnol reprend tout de suite le cours de son histoire. Le jour même de la libération de Paris, Miguel croise par hasard Estrella, jeune fille qu'il avait rencontrée sur le «Stanbrook» en fuyant Alicante. Ce qu'il advient des survivants de la Nueve est aussi brièvement évoqué. Dès le début du mois de septembre la compagnie est envoyée à Strasbourg pour participer à la campagne d'Alsace. Cette période sera particulièrement meurtrière pour la Nueve (Mesquida, 2008 : 165-166). Paris libéré, les anciens républicains espagnols pensent toujours à libérer leur pays du Franquisme mais la plupart savent aussi que les alliés n'ont pas l'intention d'entrer en Espagne contrairement à ce qui leur avait été promis. Beaucoup pensent très vite à déserter car comme le dit Miguel à son ami Granelle : «Ce n'est plus notre guerre (...) Nous ne sommes plus que de la chair à canon » (Roca, 2013a : 293). Un petit groupe décide donc de fournir des armes et des véhicules aux résistants espagnols qui luttent sur place. Miguel et d'autres chargent donc des fusils et des mitrailleuses pour les envoyer en Espagne. Paco Roca utilise le fait qu'il y ait assez peu d'informations sur les hommes de la Nueve et notamment sur Miguel Campos qui disparaît sans laisser de traces après la libération de Paris pour faire "déserter " son personnage Miguel Ruiz qui souhaite retourner libérer l'Espagne. Malheureusement, sa voiture saute sur une mine et Estrella qui l'accompagnait meurt dans l'accident. La tombe que vient régulièrement fleurir Miguel est donc celle d'Estrella. Miguel retournera en Espagne mais pour y rester peu de temps. Il retrouvera sa femme et son fils qu'il ne connaissait pas mais il se voit contraint de quitter à nouveau l'Espagne car il n'a plus d'affinités avec sa famille et il ne peut vivre dans un pays où les gens ne veulent plus lutter, lui qui s'était engagé par conviction: «Mais je me suis rendu compte que les Espagnols ne voulaient plus de guerres civiles. Le moment de lutter contre Franco était passé » (Roca, 2013a: 310). Il regrette aussi de ne pas avoir participé à l'attaque du Nid d'Aigle d'Hitler car c'est bien la Nueve et la $2^{2 \mathrm{eme}}$ DB de Leclerc qui ont pris ce bunker en mai 1945, non les troupes américaines qui sont arrivées juste après (Mesquida, 2008 : 164).

En revanche, ce qu'il advint des survivants par la suite n'est pas évoqué dans la bande dessinée. Certains, très peu, suivront Leclerc en Indochine mais il ne s'agissait plus de leur guerre, la plupart arrêteront là leur combat et leur lutte politique (Mesquida, 2008 : 167-274).

\section{Un oubli orchestré}

Comme nous l'avons signalé en introduction, les hommes de la Nueve sont des oubliés de l'Histoire française. Le cours des événements et le fait que la libération de Paris n'est pas 
l'élément décisif de la fin de la guerre (la Seconde Guerre Mondiale ne s'est pas terminée en août 1944) pourraient permettre si ce n'est d'excuser, pour le moins de comprendre l'oubli. Or, le processus se révèle simultané à l'action accomplie par les Espagnols. En effet, le 26 août un grand défilé des troupes de libération est organisé sur les Champs Élysées. La foule présente acclame les soldats de quelque nationalité qu'ils soient. De Gaulle passe en revue ses troupes et les salue toutes. Pourtant, bien qu'autorisés à défiler, les véhicules de la Nueve n'arborent plus le drapeau républicain espagnol qui a pourtant suivi les hommes depuis l'Afrique jusqu'à la veille du défilé. Les plus hautes autorités militaires ne veulent pas que les Français se rappellent que Paris a été libéré par des anarchistes et des communistes espagnols (Mesquida, 2008: 154). Il faut créer un sentiment patriotique de quelque manière que ce soit. Miguel est d'ailleurs peiné, soixante-dix ans après, par ce manque instantané de reconnaissance : « Ce qui nous a fait de la peine, c'est qu'il ait oublié le rôle joué par tous les étrangers dans la lutte pour la France libre. Espagnols, Polonais, Italiens et quelques pauvres Tchadiens » (Roca, 2013a : 277). Et à la fin de la bande dessinée avant de se séparer de Paco, il ajoute: «D'une certaine façon, nous sommes tous équitablement des oubliés de l'Histoire de France officielle. Mais nous, les Espagnols, nous étions les seuls à ne pas avoir un endroit où rentrer, après la victoire » (Roca, $2013:$ 310).

Pour le personnage de Miguel « [i]l est évident que la France devrait réécrire son histoire, à partir du moment où elle a accepté le gouvernement de Pétain pour se tirer d'affaire " (Roca, 2013 : 277). A travers ces mots il semble évident que Paco Roca exprime son propre ressenti face au manque de reconnaissance dont ont souffert ses compatriotes. Son ouvrage qui s'adresse de par sa catégorie à un large public répare cet affront. Evelyn Mesquida dont il s'est beaucoup inspiré pour créer sa bande dessinée, avait déjà commencé en 2008 ce travail de réhabilitation. Mais son ouvrage historique, bien qu'ayant bonne presse, se cantonnait aux milieux universitaires ou engagés politiquement. Il ne touchait pas suffisamment de personnes. Et comme elle le disait « la France doit aussi [comme l'Espagne] résoudre son problème de mémoire historique » visà-vis de ces hommes, étrangers pour beaucoup, qui ont combattu pour que la France soit libérée de l'emprise nazie. Le support de la bande dessinée est un très bon moyen de toucher une plus grande partie de la population car les images et le texte court sont parlants pour tout à chacun. De plus, ce média est apprécié des plus jeunes, collégiens et lycéens qui sont aussi très à l'aise avec les moyens de communication ce qui permet une diffusion de cette histoire à plus grande échelle.

\section{Conclusion}

La résistance française ne fut donc pas qu'une affaire de Français. Loin de là. Comme nous venons de le voir, des républicains espagnols ont mis de côté une partie de leurs convictions pour rendre sa liberté à la France en s'engageant dans une armée nationale, celle de la France libre. Pourtant, durant de trop nombreuses années, leur courage et leur abnégation n'ont jamais été reconnus. Il faudra attendre le 24 août 2014 pour qu'ait lieu une commémoration de réelle importance, soit 70 ans après les faits.

De plus, cette reconnaissance tardive est le fruit des travaux d'une espagnole et non des autorités françaises, Évelyn Mesquida. C'est elle qui, en 2008, lors de la promulgation de la Loi de Mémoire Historique, entreprend de réhabiliter la mémoire de ces personnes. Son ouvrage, qui est la référence de Paco Roca, permet au graphiste espagnol de nous livrer 
un témoignage bouleversant sur l'histoire de ces hommes, des étrangers qui se sont sacrifiés pour libérer Paris des troupes nazies. Nous suivons leur parcours depuis la fuite d'Espagne, en passant par les camps d'Afrique jusqu'au défilé triomphal sur les ChampsÉlysées. Cette bande dessinée illustre avec brio les souffrances et le ressentiment de ces hommes mais aussi leur combat pour la liberté et la lutte pour leurs idées jusqu'à la mort ou dans la vieillesse.

Mais la réhabilitation ne peut s'arrêter là. Il reste encore beaucoup de chemin à parcourir pour que ces hommes, quelle que soit leur nationalité, soient réellement reconnus comme des anciens combattants pour la France.

\section{BIBLIOGRAPHIE}

ROCA, Paco (2013). La Nueve. Les républicains espagnols qui ont libéré. Paris : Delcourt.

ROCA, Paco (2013). Los surcos del azar. Espagne : Atisberri.

ARMAND-DREYFUS, Geneviève (1999). L'exil des républicains espagnols en France. De la guerre civile à la mort de Franco. Paris : Albin Michel.

LEVISSE-TOUZE, Christine (2008). Dictionnaire historique de la résistance, sous la direction de François MARCOT. Paris : éditions Robert Laffont, « collection Bouquins ».

MESQUIDA, Évelyn (2008). La Nueve. Los españoles que liberaron París. Espagne : Ediciones B.

VIEL, Jacques. Interview réalisée pour les éditions Delcourt. [En ligne] (actualisé le $1^{\mathrm{er}}$ mai 2014)

[disponible le 28/03/15]

URL : http://www.youtube.com/watch?v=YimsltYqF1Q

GONZALEZ HARBOUR, Berna (2009). « Entrevista con Evelyn Mesquida, Francia debe resolver su memoria histórica » [En ligne], El País, 13/07/2009 [disponible le 28/03/2015]

URL : http://elpais.com/diario/2009/07/13/ultima/1247436002_850215.html

\section{RÉSUMÉS}

La Seconde Guerre Mondiale est un évènement prolifique en films, livres et autres supports. Pourtant, malgré les nombreux ouvrages (de fiction ou non) produits et publiés, tout n'a pas encore été dit et révélé sur cet épisode oh combien important de l'Histoire. Nous nous proposons donc d'analyser la bande dessinée de Paco Roca, auteur contemporain espagnol, qui dans son œuvre La Nueve. Les républicains espagnols qui ont libéré Paris relate l'histoire vraie des exilés républicains espagnols qui ont rejoint les forces françaises de la résistance au nazisme et au gouvernement de Pétain et ont participé à la libération de la capitale française. Ce roman graphique permet de connaitre leur parcours pour le moins incroyable depuis leur fuite d'une Espagne entièrement soumise au joug franquiste depuis le $1^{\mathrm{er}}$ avril 1939. Il est aussi l'occasion de rendre hommage à ces hommes oubliés de l'Histoire. 
The Second World War has become the topic of movies, books and other formats. Although many works (fictional or not) have been shot or published, not everything has been said or revealed about this very important event in History. Therefore, we are going to study Paco Roca's comic strip La Nueve. Les républicains espagnols qui ont libéré Paris. The contemporary Spanish author tels the true story of republican Spanish refugees who joined the French Resistance against the Nazi German occupation of France and the Vichy Regime and who took part in the liberation of the French capital city. This graphic novel tells us about their incredible route from their escape of Nationalist Spain in early 1939. The novel gives the opportunity to pay tribute to these men who have entirely been forgotten by History.

\section{INDEX}

Mots-clés : bande dessinée, espagnols, Résistance, Libération, Paris, hommage

Keywords : comic strip, Spanish, Resistance, Liberation, Paris, tribute

\section{AUTEUR}

\section{VANESSA AUROY}

Université d'Angers

vanessa.auroy@etud.univ-angers.fr 